\title{
Tissue and plasma putrescine levels in non-survivors of sepsis in a fluid-resuscitated rat model of faecal peritonitis
}

\author{
DT Andreis ${ }^{1,2^{*}}$, W Khaliq ${ }^{1}$, S Neugebauer ${ }^{3}$, M Kiehntopf $^{3}$, M Singer $^{1}$ \\ From ESICM LIVES 2015 \\ Berlin, Germany. 3-7 October 2015
}

\section{Introduction}

The polyamine, putrescine, was first isolated from putrefying meat but is thought to play an important role in cell growth and differentiation ([1]). It also generates succinate via GABA and can thus serve as an energy source to the small intestine ([2]). Elevated plasma putrescine levels have been reported in an endotoxic rodent model ([3]). We have previously characterized a $72 \mathrm{~h}$ fluid-resuscitated rat model of faecal peritonitis where prognostication can be accurately made as early as $6 \mathrm{~h}$ post-insult ([4]).

\section{Objectives}

Using this long-term sepsis model, to assess differences in liver and plasma levels of putrescine in predicted survivors and non-survivors.

\section{Methods}

Awake, instrumented yet fully mobile male Wistar rats $(325 \pm 15 \mathrm{~g})$ received an i.p. injection of $4 \mu \mathrm{l} / \mathrm{g}$ faecal slurry. Fluid resuscitation (50:50 mix of $5 \%$ glucose/ Hartmann's; $10 \mathrm{ml} / \mathrm{kg} / \mathrm{h}$ ) was commenced at $2 \mathrm{~h}$. At $6 \mathrm{~h}$, an echo-measured heart rate cut-off of $460 \mathrm{bpm}$ was used to classify animals into predicted survivors or non-survivors. Animals were sacrificed at $6 \mathrm{~h}, 24 \mathrm{~h}$ or $72 \mathrm{~h}$ for liver and blood sampling. A group of control animals were treated identically but without injection of faecal slurry. Putrescine levels were measured using mass spectrometry. Results were analysed using two-way ANOVA and post-hoc testing and considered statistically significant when $\mathrm{p}<0.05$.

University College London, Bloomsbury Institute of Intensive Care Medicine, London, United Kingdom

Full list of author information is available at the end of the article
Table 1

\begin{tabular}{|c|c|c|c|c|}
\hline \multicolumn{2}{|c|}{$\begin{array}{c}\text { Putrescine } \\
\text { level }(\mu \mathrm{mol} / \mathrm{L})\end{array}$} & \multirow{2}{*}{$\begin{array}{c}\text { Control } \\
1.59 \pm 0.20\end{array}$} & \multirow{2}{*}{$\begin{array}{c}\text { Septic Survivors } \\
1.91 \pm 0.24\end{array}$} & \multirow{2}{*}{$\begin{array}{c}\text { Septic Non-Survivors } \\
3.51 \pm 0.42^{*}\end{array}$} \\
\hline $6 \mathrm{~h}$ & Plasma & & & \\
\hline & Liver & $0.29 \pm 0.06$ & $0.41 \pm 0.13$ & $3.58 \pm 0.63^{*}$ \\
\hline \multirow[t]{2}{*}{$24 \mathrm{~h}$} & Plasma & $1.39 \pm 0.10$ & $1.68 \pm 0.17$ & $4.20 \pm 0.82^{*}$ \\
\hline & Liver & $0.44 \pm 0.21$ & $0.42 \pm 0.16$ & $3.61 \pm 1.45^{*}$ \\
\hline \multirow[t]{2}{*}{$72 \mathrm{~h}$} & Plasma & $1.28 \pm 0.09$ & $1.20 \pm 0.12$ & - \\
\hline & Liver & $0.47 \pm 0.10$ & $0.31 \pm 0.09$ & - \\
\hline \multicolumn{5}{|c|}{ Data shown as median $\pm S E ; * p<0.05$} \\
\hline
\end{tabular}

\section{Results}

In this model septic animals had a mortality rate of $56 \%$ with death occurring between 18-36 h. At $6 \mathrm{~h}$ septic animals displayed only mild clinical features of illness. However, even as early as $6 \mathrm{~h}$, significant differences were noted in putrescine levels in liver and plasma from non-surviving septic animals.

\section{Conclusions}

An association was seen between eventual non-survival and elevated putrescine levels in both liver and plasma at both $6 \mathrm{~h}$ and $24 \mathrm{~h}$. The significance of this finding warrants further investigation.

\section{Grant Acknowledgement}

ESICM Basic Science Award, Intensive Care Foundation (UK), NIHR

\footnotetext{
Authors' details

'University College London, Bloomsbury Institute of Intensive Care Medicine, London, United Kingdom. ${ }^{2}$ Università degli Studi di Milano, Dipartimento di Fisiopatologia Medico-Chirurgica e dei Trapianti, Milan, Italy. ${ }^{3}$ University Hospital Jena, Institut für Klinische Chemie und Laboratoriumsdiagnostik, Jena, Germany.
}

(c) 2015 Andreis et al.; This is an Open Access article distributed under the terms of the Creative Commons Attribution License (http:// creativecommons.org/licenses/by/4.0), which permits unrestricted use, distribution, and reproduction in any medium, provided the original work is properly cited. 


\section{References}

1. Tabor CW, et al: Ann Rev Biochem 1984, 53:747-90.

2. Bardocz S, et al: Gut 1998, 42:24-48.

3. Lortie MJ, et al: Am J Physiol - Cell Physiol 2000, 278:C1191-9.

4. Rudiger A, et al: Clin Sci 2013, 124:391-401.

doi:10.1186/2197-425X-3-S1-A616

Cite this article as: Andreis et al:: Tissue and plasma putrescine levels in

non-survivors of sepsis in a fluid-resuscitated rat model of faecal peritonitis.

Intensive Care Medicine Experimental 2015 3(Suppl 1):A616.

\section{Submit your manuscript to a SpringerOpen ${ }^{\mathcal{O}}$ journal and benefit from:}

- Convenient online submission

- Rigorous peer review

- Immediate publication on acceptance

- Open access: articles freely available online

- High visibility within the field

- Retaining the copyright to your article

Submit your next manuscript at $>$ springeropen.com 